\section{Re: The So-Called Stunning of Thyroid Tissue}

TO THE EDITOR: In a recent report, Sisson et al. (1) hypothesized that so-called stunning of thyroid tissue results from the early effects of the ablation dose of ${ }^{131} \mathrm{I}$ in the form of accelerated disappearance of the therapeutic radionuclide from thyroid tissue. It was argued that initial uptake of the therapeutic dose within the first day (determined in their study by extrapolation of observed data back to time zero) was not impaired by the diagnostic dose of ${ }^{131} \mathrm{I}$. So-called stunning - that is, reduced uptake of the therapeutic dose observed from day 1 onwardresults from more rapid clearance of the therapeutic radioiodine than of the diagnostic radioiodine. This hypothesis is somewhat at variance with our postulate (2) that the process of ablation begins with radiation damage to the iodine uptake mechanism of the cells within the thyroid remnant. In other words, there is a degree of self-stunning as the therapeutic radionuclide is being taken up by thyroid tissue, leading to a relative reduction in initial uptake.

Their hypothesis ( 1 ) is based on observations on 10 patients in whom the time course of radionuclide in thyroid tissue was studied after the administration of diagnostic and therapeutic ${ }^{131}$ I. The intention was to measure thyroid uptake of both diagnostic $(37 \mathrm{MBq}[1 \mathrm{mCi}])$ and therapeutic $(1,110 \mathrm{MBq}[30$ $\mathrm{mCi}]$ or $5,550 \mathrm{MBq}[150 \mathrm{mCi}])$ radionuclide daily for the first $7 \mathrm{~d}$. In fact, this daily measurement was achieved in only 3 patients because of difficulties with patient compliance after administration of the diagnostic dose. Visual inspection of their data indicates that in 2 of this small subgroup of patients, clearance of the therapeutic radionuclide was indeed more rapid. In the third patient, the clearance curves for both doses of radionuclide were similar. In the remaining 7 patients, the data were insufficient to draw any conclusions on the comparative clearance of ${ }^{131} \mathrm{I}$ after administration of diagnostic and therapeutic radionuclide.

However, the main basis for their hypothesis is their model of iodine kinetics for an ablative dose of radioiodine. In 5 patients, the disappearance of ${ }^{131}$ I was biexponential, with a rapid first phase, but the fact that only 5 data points were used (from either day 1 or day 2 onward) imposes some uncertainty on the derived curve parameters. Furthermore, 2 outlier points were excluded in 2 of the 5 patients. In the remaining patients, the disappearance was either monoexponential or followed an unusual pattern of late acceleration. The best-fit curves were then extrapolated back to time zero, and a "reasonable assumption" was made that the "near-maximum uptakes of radioiodine are between 6 and $24 \mathrm{~h}$ after ingestion." It would be interesting to know the basis for this assumption. In normal thyroid tissue, the half-time of iodine uptake is usually taken to be $8 \mathrm{~h}$ (3), implying that maximal uptake does not occur before $24 \mathrm{~h}$, albeit in the presence of normal thyroid-stimulating hormone levels.

The limited diagnostic uptake data (only 2 data points in 7/10 patients) were also extrapolated back to time zero, and therapeutic or diagnostic uptake ratios were calculated at 2, 1, 0.25, and $0 \mathrm{~d}$, the mean ratios at these time points being $0.65,1.01,1.63$, and

COPYRIGHT ( 2007 by the Society of Nuclear Medicine, Inc.
2.26, respectively. From analysis of these data, the authors (1) concluded that only 1 patient showed a pattern that could reflect a substantial impairment of uptake of ${ }^{131} \mathrm{I}$ by thyroid tissue. An alternative conclusion from their published data is that, compared with diagnostic radioiodine, therapeutic radioiodine showed unexpectedly enhanced uptake in 5 of 10 patients. This conclusion raises doubts about the validity of their assumption with respect to the time of maximal uptake and the use of limited data for extrapolation purposes.

In our work (2), we quantitatively compared ${ }^{131} \mathrm{I}$ and ${ }^{123} \mathrm{I}$ as diagnostic agents. Our results in the case of ${ }^{131} \mathrm{I}$ were broadly similar to those reported by Sisson et al. (1) in that the thyroid content of therapeutic radioiodine (a 4,000-MBq [108-mCi] dose) was substantially less that that of diagnostic radioiodine (a 120$\mathrm{MBq}$ [3.2-mCi] dose) 1-3 d after dose administration. In the case of ${ }^{123} \mathrm{I}$, we compared the uptake of a diagnostic dose of ${ }^{131} \mathrm{I}$ (200 $\mathrm{MBq}[5.4 \mathrm{mCi}])$ and an ablative dose of ${ }^{131} \mathrm{I}(4,000 \mathrm{MBq}$ [108 $\mathrm{mCi}]$ ) at $24 \mathrm{~h}$ after administration in 16 patients. We took care to ascertain the response of our $\gamma$-camera system to high counting rates and to allow for the different absorption characteristics of photon emissions from the 2 radionuclides. For measurement of therapeutic uptake, we preset the $\gamma$-camera to a high counting-rate mode and corrected for any count losses, as detailed in our paper. Some other camera systems do not allow the operator to preset a high counting-rate mode; the system automatically switches over depending on counting rate, potentially leading to anomalies in serial studies of uptake, in which the counting rate is decreasing daily. Sisson et al. (1) give little detail on the counting-rate response of the $\gamma$-camera systems used in their study.

The results for ${ }^{123}$ I were unexpected in that stunning was still observed, albeit to a lesser extent than with ${ }^{131} \mathrm{I}$ (58.8\% and $32.8 \%$, respectively, for the ratio of median therapeutic uptake to diagnostic uptake). This finding led us to conclude that the ablative dose itself, on being taken up by thyroid tissue, caused radiation damage to the iodine-trapping function of the cells, thus inhibiting uptake. We calculated that a substantial radiation dose could be delivered to thyroid tissue within the first $8 \mathrm{~h}$ of administration of the ablative radioiodine. Comparison of 2 uptake measurements (\% dose) 1-3 d after therapy in 15 patients revealed some loss of radionuclide due to biologic turnover, but the result did not achieve statistical significance in the group as a whole. It is difficult to compare this finding with the data published by Sisson et al. (1) because their disappearance curves included radioactive decay.

Both papers raise the possibility that so-called stunning is partly due to injury to thyroid tissue by the ablative dose. We suggested that the observed reduction in uptake is due in part to early damage to the iodine uptake process by the ablative dose (2), whereas Sisson et al. have suggested that the primary cause of "stunning" is early destruction of thyroid tissue (1). In their hypothesis, initial uptake of the ablative dose is not impaired.

\section{REFERENCES}

1. Sisson JC, Avram AM, Lawson SA, Gauger PG, Doherty GM. The so-called stunning of thyroid tissue. J Nucl Med. 2006;47:1406-1412. 
2. Hilditch TE, Dempsey MF, Bolster AA, McMenemin RM, Reed NS. Self-stunning in thyroid ablation: evidence from comparative studies of diagnostic ${ }^{131} \mathrm{I}$ and ${ }^{123} \mathrm{I}$. Eur J Nucl Med Mol Imaging. 2002;29: 783-788.

3. International Commission on Radiological Protection (ICRP). Radiation Dose to Patients from Radiopharmaceuticals. Oxford, U.K.: Pergamon Press; 1987. Publication 53.

Thomas E. Hilditch Alison A. Bolster Mary F. Dempsey Nick S. Reed Greater Glasgow and Clyde Health Board Glasgow, United Kingdom

DOI: 10.2967/jnumed.106.037788

REPLY: In the review of the literature for our paper (1), we overlooked the report by Hilditch et al. (2). We regret this omission.

The letter by Hilditch et al. makes a case for some stunning effects from diagnostic doses of ${ }^{131}$ I. However, points in the letter and in their paper (2) leave uncertainties.

Several differences in methods may have contributed to the differences between our results and those of Hilditch et al. We administered therapeutic ${ }^{131} \mathrm{I}$ immediately after the last measurements of diagnostic radioiodine. In the Hilditch communication, there was an interval of 3-38 d between the end of diagnostic testing and ingestion of the therapeutic dose of ${ }^{131} \mathrm{I}$. The effects of prolonged stimulation by thyroid-stimulating hormone are unknown. For diagnostic ${ }^{131} \mathrm{I}$, we administered $37 \mathrm{MBq}(1 \mathrm{mCi})$, in contrast to the $120 \mathrm{MBq}(3.2 \mathrm{mCi})$ administered by Hilditch et al. The consequences of the differences in diagnostic dose are unknown.

Hilditch et al. reported that when ${ }^{123}$ I was the diagnostic agent, the therapeutic-to-diagnostic ratio was $58.8 \%$. To account for this ratio, a substantial loss of therapeutic radioactivity must have occurred between day 1 (the time when ${ }^{123}$ I was measured) and day 3 after the therapeutic dose of ${ }^{131} \mathrm{I}$. The report also stated that, in some patients, ${ }^{131}$ I was measured a second time during the $1-3 \mathrm{~d}$ after treatment. No individual data points were recorded, but the mean of the differences between the 2 measurements was found to be insignificant. Yet, the obligatory loss of radioactivity was unlikely to have been instantaneous; the loss at the earlier time should have been less than the loss at $3 \mathrm{~d}$. Thus, their assays of radioactivity must be associated with uncertainty.

In the Hilditch paper, the therapeutic-to-diagnostic ratio after diagnostic doses of ${ }^{123} \mathrm{I}(58.8 \%)$ and ${ }^{131} \mathrm{I}$ (32.8\%) were significantly different. But after diagnostic ${ }^{123} \mathrm{I}$ and ${ }^{131} \mathrm{I}$, both ranges for the therapeutic-to-diagnostic ratios varied considerably (17\%-130\% and $6 \%-93 \%$, respectively) and overlapped appreciably. The possibility exists that most of the decline in the ratios of both groups arose from the effects of the treatment doses.

With regard to our technique for acquiring data from high levels of radioactivity, linearity existed at the counting rates observed even after the largest treatment doses in our patients. We acknowledged the limitations in the applications of our measurements. However, the general pattern of our results suggested that much, if not all, of the differences between diagnostic and therapeutic concentrations of ${ }^{131} \mathrm{I}$ in thyroid tissue result from the radiation injury inflicted by the treatment dose, at least when the diagnostic dose was $37 \mathrm{MBq}$.

\section{REFERENCES}

1. Sisson JC, Avram AM, Lawson SA, Gauger PG, Doherty GM. The so-called stunning of thyroid tissue. J Nucl Med. 2006;47:1406-1412.

2. Hilditch TE, Dempsey MF, Bolster AA, McMenemin RM, Reed NS. Selfstunning in thyroid ablation: evidence from comparative studies of diagnostic ${ }^{131} \mathrm{I}$ and ${ }^{123}$ I. Eur J Nucl Med Mol Imaging. 2002;29:783-788.

\section{James C. Sisson \\ University of Michigan Heath Systems Ann Arbor, Michigan}

DOI: 10.2967/jnumed.106.038497 